\title{
APTIDÃO DA BACIA DO RIO DOURADOS PARA O CULTIVO DE ALGUMAS ESPÉCIES DE EUCALIPTOS
}

\author{
Marco Aurélio Carneiro*, Omar Daniel**, Antonio Carlos Tadeu Vitorino***, Éder Comunello**** \\ *Eng. Agrônomo, M.Sc. - marco999@ubbi.com.br \\ **Eng. Florestal, Dr., Depto. de Ciências Agrárias, UFMS - omard@ceud.ufms.com.br \\ ***Engenheiro Agrônomo, Dr., Depto. de Ciências Agrárias, UFMS - vitorino@ceud.ufms.com.br \\ ****Engenheiro Agrônomo, M.Sc., Embrapa Agropecuária Oeste - eder@cpao.embrapa.br \\ Recebido para publicação: 07/06/2006 - Aceito para publicação: 21/09/2006
}

\begin{abstract}
Resumo
O objetivo desse trabalho foi a geração de um mapa de aptidão florestal considerando níveis de manejo para Eucalyptus camaldulensis Dehn, Eucalyptus citriodora Hook, Eucalyptus grandis W. Hill ex Maiden e Eucalyptus urophylla St. Blake na bacia do rio Dourados, com auxílio do software SPRING 4.1/INPE. A área de estudo localiza-se no estado de Mato Grosso do Sul, entre as coordenadas $21^{\circ} 56^{\prime} \mathrm{S}-22^{\circ} 38^{\prime} \mathrm{S}$ e $53^{\circ} 57^{\prime} \mathrm{O}-5^{\circ} 57^{\prime} \mathrm{O}$, com precipitação média anual na região em torno de $1.400 \mathrm{~mm}$ e clima do tipo Cwa. Os solos são predominantemente da classe Latossolos, ocorrendo também Argissolos, Neossolos Quartzarênicos e Gleissolos em menores proporções. As exigências edafoclimáticas das espécies citadas foram baseadas na sua ocorrência nas regiões de origem. A partir do cruzamento dos mapas de solos, altimetria, hidrografia, declividade, áreas de preservação permanente e de uso da terra, e considerando-se as restrições para cada espécie, geraramse três mapas: um para E. urophylla, outro para E. camaldulensis, E. citriodora e E. grandis e um terceiro considerando os níveis de manejo tecnológico. Como resultado, constatou-se que mais de $87 \%$ (excetuando-se a reserva legal) da área da bacia do rio Dourados é apta para o cultivo das espécies consideradas, podendo-se contar com sete classes de manejo tecnológico.

Palavras-chave: Eucalyptus; bacia hidrográfica; geoprocessamento; zoneamento.
\end{abstract}

\begin{abstract}
Land aptitude of Dourados River's Basin for some species of eucalyptus crops. The aim of this work was to generate Land Aptitude Maps for Eucalyptus camaldulensis Dehn, Eucalyptus citriodora Hook, Eucalyptus grandis W. Hill ex Maiden and Eucalyptus urophylla St. Blake in Dourados River's Basin by using Geographic Information System (GIS). The geographical area of study is located in Mato Grosso do Sul State between $21^{\circ} 56^{\prime} \mathrm{S}-22^{\circ} 38^{\prime} \mathrm{S}$ and $53^{\circ} 57^{\prime} \mathrm{W}-55^{\circ} 57^{\prime} \mathrm{W}$. The medium annual rainfall in this area is about $1.400 \mathrm{~mm}$ and Cwa type climate. The soils are "Latossolos", some "Argissolos", "Neossolos Quartzarenicos" and "Gleissolos". The environmental requirements of these species of Eucalyptus were based on their development in their place of origin according to the bibliography. Maps of soils, altimetry, hydrography, declivity, permanent buffer area and land use have been used to define a database system through the Brazilian software SPRING 4.1/INPE. Three maps were generated, due to the restrictions considered: one map of E. urophylla, another map of E. camaldulensis, E. citriodora and E. grandis and a third one considering management levels. As a result, more than $87 \%$ (except the legal reserve) of Dourados River's Basin is promising for the development of the above mentioned species, considering seven management levels.
\end{abstract}

Keywords: Eucalyptus; watershed; GIS; zoning.

\section{INTRODUÇÃO}

Dentro do cenário socioeconômico regional, a bacia hidrográfica do rio Dourados ocupa uma área de grande importância. Constituída por um alto percentual de solos com boa aptidão agrícola, tem na agricultura e na pecuária suas principais bases econômicas (MATO GROSSO DO SUL, 2000). A ocupação desordenada das terras tem agravado a situação ambiental da região, como demonstra o estudo 
de Daniel et al. (2002), no qual são apresentadas taxas totais de vegetação nativa inferiores a $18 \%$ do total da área.

Nos últimos anos, a atividade florestal regional tem sido implementada especialmente com a introdução de eucalipto, baseada em quatro espécies (Eucalyptus camaldulensis, E. citriodora, E. grandis, E. urophylla), sendo as duas últimas as mais plantadas. Essa escolha tem como uma das justificativas a dificuldade em se optar por outras espécies em que se tenha a segurança de sua adaptação. São raros os trabalhos de pesquisa com procedências e progênies que procuraram definir espécies a serem cultivadas, como, por exemplo, Daniel (1998).

A implantação aleatória de povoamentos florestais e a relativa falta de informações sobre o cultivo do eucalipto na região revelam a necessidade de maiores estudos a esse respeito, justificando um zoneamento para aptidão baseado nas condições dos locais de origem do gênero.

Outra justificativa para estudos dessa natureza refere-se à oferta de madeira de eucalipto. $\mathrm{O}$ Estado de MS tem hoje cerca de 113 mil hectares com plantios do gênero, dos quais aproximadamente 93\% já estão de alguma forma comprometidos com o comércio. A oferta de madeira de eucalipto no Estado já é praticamente inexistente, e o volume remanescente, persistindo a tendência atual, estará exaurido dentro de 3 a 4 anos (RAMIRES JÚNIOR, 2003).

O zoneamento para aptidão deve prever a necessidade de decisões políticas e qualitativas que considere as questões ambientais e legais para a implantação do sistema de produção mais adequado, sem com isso se recomendar a substituição das matas restantes ou das áreas de lavoura pela cultura do eucalipto.

Essa primeira aproximação de zoneamento leva em conta principalmente as condições edafoclimáticas dos locais de origem de algumas espécies do gênero Eucalyptus, considerando também diversos níveis de manejo, de acordo com as unidades de solos encontradas na região. As bases de dados foram organizadas com a utilização de um Sistema de Informações Geográficas (SIG).

Os SIG's apresentam-se como ferramentas poderosas para esse fim, por trabalharem com uma grande variedade e quantidade de dados em tempo relativamente curto, sem perder a qualidade, quando comparados com procedimentos tradicionais mais demorados.

Dentre os SIG's existentes, optou-se pelo sistema SPRING (Sistema para Processamento de Informações Georreferenciadas), desenvolvido pelo INPE (Instituto Nacional de Pesquisas Espaciais), que pode ser definido como um "conjunto de ferramentas voltadas à coleta e tratamento de informações espaciais, além da geração de saídas na forma de mapas convencionais, relatórios, arquivos digitais e outros, devendo prover recursos para armazenamento, gerenciamento, manipulação e análise de dados" (CÂMARA et al., 1996).

O objetivo deste trabalho, portanto, foi a geração de um mapa de aptidão florestal considerando níveis de manejo para Eucalyptus camaldulensis, Eucalyptus citriodora, Eucalyptus grandis e Eucalyptus urophylla na bacia do rio Dourados, com o auxílio de um Sistema de Informações Geográficas.

\section{MATERIAL E MÉTODOS}

\section{Área de trabalho}

A bacia do rio Dourados localiza-se na porção sul do estado do Mato Grosso do Sul, entre as coordenadas geográficas $21^{\circ} 56^{\prime} \mathrm{S}-22^{\circ} 38^{\prime} \mathrm{S}$ e $53^{\circ} 59^{\prime} \mathrm{O}-55^{\circ} 57^{\prime} \mathrm{O}$ (Figura 1), abrangendo total ou parcialmente onze municípios. Situa-se na bacia do rio Ivinhema, que, por sua vez, se insere na bacia hidrográfica do rio Paraná (MATO GROSSO DO SUL, 2000).

O clima na região é do tipo Cwa (clima temperado úmido, com inverno seco e verão quente). Dados gerados na estação meteorológica da Embrapa Agropecuária Oeste são apresentados na tabela 1, e os valores de déficit hídrico máximo esperado, na figura 2 (FIETZ, 2001). A altitude da bacia se aproxima dos $200 \mathrm{~m}$ nas proximidades da foz do Rio Dourados, alcançando cerca de $700 \mathrm{~m}$ na sua cabeceira no município de Antônio João (DANIEL et al., 2002).

Os Latossolos representam mais de 97\% do total da área da bacia do rio Dourados; os Argissolos ocupam 2,14\%; os Gleissolos ocupam 0,30\%; os Neossolos Quartzarênicos, apenas 0,13\% (URCHEI, 2001). Na tabela 2 são apresentadas as principais unidades de solos encontradas na bacia do rio Dourados. 
Tabela 1. Precipitação (P), temperatura do ar (T) e umidade relativa (UR) de Dourados (médias anuais do período de 1979 a 1999).

Table 1. Dourados' rainfall data $(\mathrm{P})$, mean air temperature $(\mathrm{T})$ and relative humidity (UR) (annual averages from 1979/1999 period).

\begin{tabular}{cccccccccccccc}
\hline & Jan & Fev & Mar & Abr & Mai & Jun & Jul & Ago & Set & Out & Nov & Dez $\begin{array}{c}\text { Total/ } \\
\text { Média } \\
\text { anual }\end{array}$ \\
\hline $\mathrm{P}(\mathrm{mm})$ & 156,4 & 123,7 & 151,1 & 120,2 & 115,0 & 75,9 & 38,1 & 48,7 & 105,5 & 147,2 & 150,7 & 176,5 & 1.409 \\
$\mathrm{~T}\left({ }^{\circ} \mathrm{C}\right)$ & 25,3 & 24,6 & 24,4 & 22,7 & 19,7 & 17,6 & 17,7 & 19,8 & 21,1 & 23,5 & 24,6 & 25,2 & 22,2 \\
$\mathrm{UR}(\%)$ & 81 & 83 & 80 & 79 & 80 & 80 & 73 & 69 & 70 & 72 & 73 & 77 & 76 \\
\hline Fonte: Fietz, 2001 & & & & & & & & & & & &
\end{tabular}
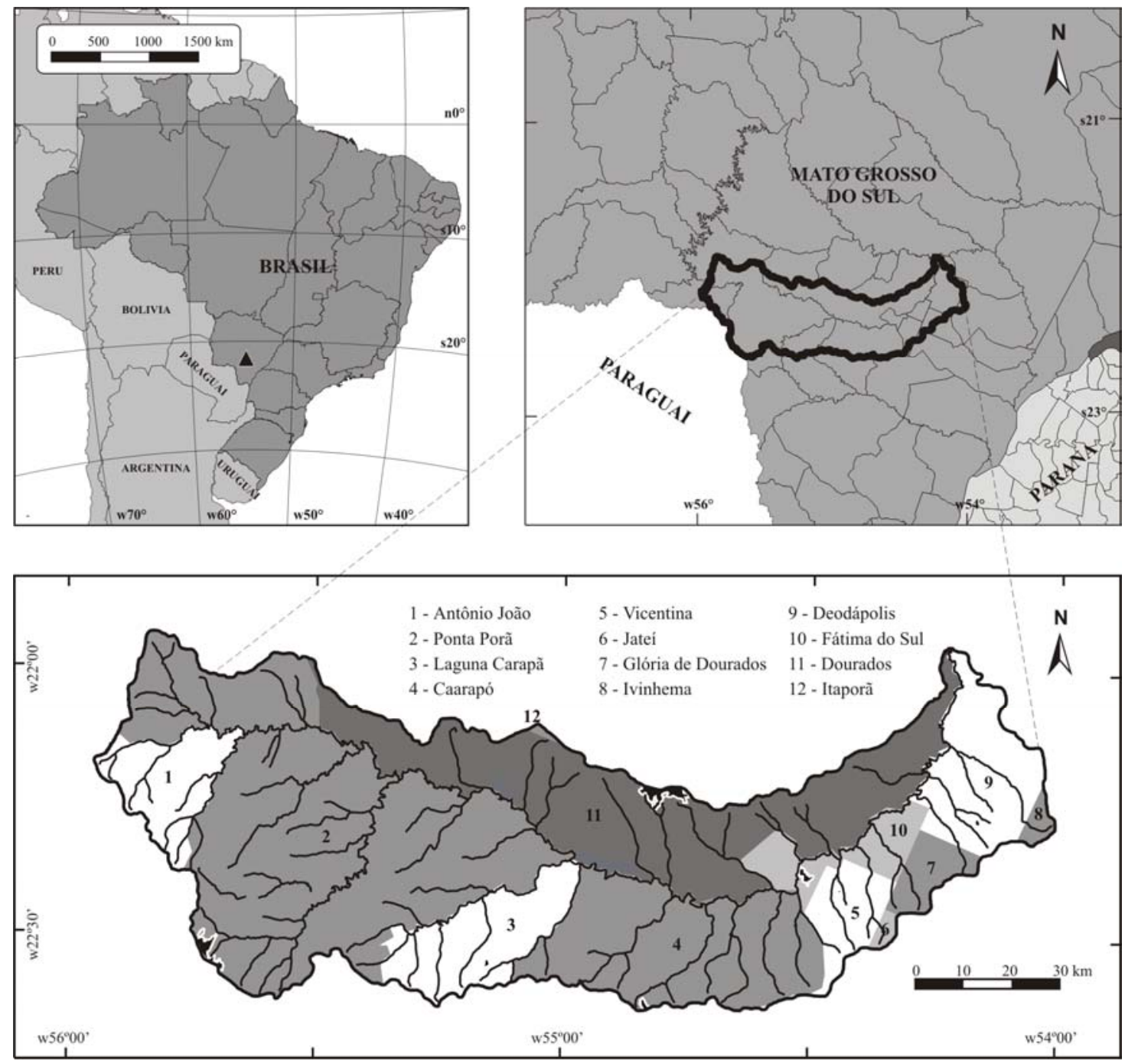

Figura 1. Localização da área de estudo.

Figure 1. Work area location. 


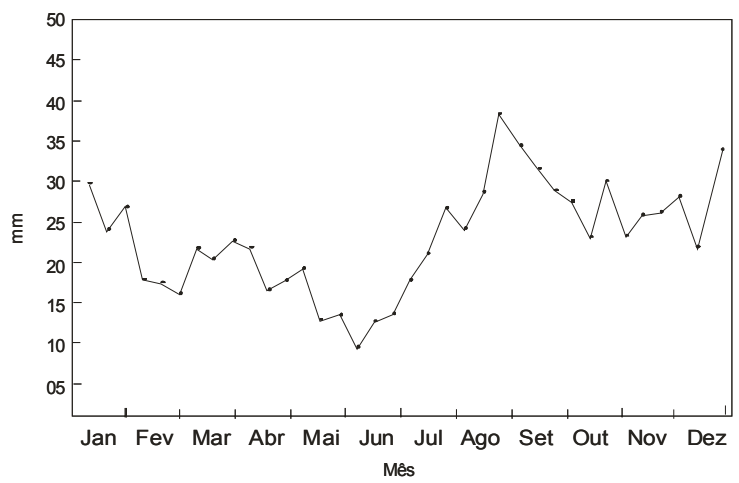

Figura 2. Déficit hídrico decendial máximo esperado na região de Dourados para o período de retorno de 4 anos ou $75 \%$ de probabilidade. Período de junho de 1979 a dezembro de 1998. Fonte: Fietz, 2001.

Figure 2. Maximum estimated decendial water deficit in Dourados region for the return period of 4 years or $75 \%$ of probability. June 1979 to December 1998. Fonte: Fietz, 2001.

Tabela 2. Unidades de solo presentes na bacia do rio Dourados (1/250.000), extraída da legenda da carta de solos do estado de Mato Grosso do Sul (SEPLAN, 1990).

Table 2. Soil classes within Dourados River's Basin, taken from the soil map legend of the State of Mato Grosso do Sul (SEPLAN, 1990).

\begin{tabular}{|c|c|}
\hline Solo & Características do solo \\
\hline$\overline{\mathrm{AQa}^{1}}{ }^{1}$ & Areia Quartzosa álica plano e levemente ondulado. \\
\hline $\mathrm{HGPe} 7^{2}$ & $\begin{array}{l}\text { Glei Pouco Húmico eutrófico, argila de alta atividade, textura argilosa + Plintossolo eutrófico, argila de alta } \\
\text { atividade, textura franca horizonte A, argilosa horizonte B + Glei Húmico eutrófico, argila de alta atividade, } \\
\text { textura argilosa. }\end{array}$ \\
\hline $\mathrm{LEa}^{3}$ & Latossolo Vermelho-Escuro álico, textura argilosa, plano. \\
\hline $\mathrm{LEa} 2^{3}$ & Latossolo Vermelho-Escuro álico, textura argilosa, plano e suavemente ondulado. \\
\hline $\mathrm{LEa}^{3}$ & Latossolo Vermelho-Escuro álico e distrófico, textura franca, plano e suavemente ondulado. \\
\hline $\mathrm{LEa} 11^{3}$ & Latossolo Vermelho-Escuro álico, textura franca, plano e suavemente ondulado + Areias Quartzosas álica. \\
\hline LEa12 $2^{3}$ & Latossolo Vermelho-Escuro álico, textura franca, suavemente ondulado e plano + Areias Quartzosas álica. \\
\hline LEa15 $5^{3}$ & $\begin{array}{l}\text { Latossolo Vermelho-Escuro álico, textura argilosa, plano + Glei Pouco Húmico álico, argila de baixa } \\
\text { atividade, textura argilosa + Plintossolo álico, argila de baixa atividade, textura franca horizonte A, argilosa } \\
\text { horizonte B. }\end{array}$ \\
\hline $\mathrm{LEa} 20^{3}$ & $\begin{array}{l}\text { Latossolo Vermelho-Escuro álico, textura franca, plano e suavemente ondulado + Latossolo Vermelho } \\
\text { Escuro álico, textura franca, muito pedregoso, suavemente ondulado + Latossolo Vermelho-Amarelo álico, } \\
\text { textura franca, plano e suavemente ondulado. }\end{array}$ \\
\hline $\mathrm{LRa1}^{3}$ & Latossolo Roxo álico, textura muito argilosa, plano. \\
\hline $\mathrm{LRa}^{3}$ & Latossolo Roxo álico, textura muito argilosa plano e suavemente ondulado. \\
\hline LRd $5^{3}$ & $\begin{array}{l}\text { Latossolo Roxo distrófico, horizonte A moderado e proeminente, textura argilosa e muito argilosa, } \\
\text { suavemente ondulado + Terra Roxa Estruturada eutrófica, pouco profundo não pedregoso e pedregos, } \\
\text { horizonte A chernozêmico, textura argilosa e muito argilosa, plano e suavemente ondulado + Glei Pouco } \\
\text { Húmico eutrófico, argila de baixa atividade, textura argilosa, plano. }\end{array}$ \\
\hline $\mathrm{LRd} 7^{3}$ & $\begin{array}{l}\text { Latossolo Roxo distrófico e eutrófico, textura muito argilosa e argilosa, suavemente ondulado + Glei Pouco } \\
\text { Húmico eutrófico, argila de alta atividade, textura argilosa, plano + Glei Húmico eutrófico, textura argilosa, } \\
\text { argila de alta atividade + Plintossolo eutrófico, argila de alta atividade, textura franca, horizonte A argiloso, } \\
\text { horizonte B abrúptico + Latossolo Roxo eutrófico pouco profundo, argiloso, suavemente ondulado. }\end{array}$ \\
\hline $\operatorname{LRe}^{3}$ & Latossolo Roxo eutrófico e distrófico, textura muito argilosa, plano e suavemente ondulado. \\
\hline $\operatorname{LRe}^{3}$ & $\begin{array}{l}\text { Latossolo Roxo eutrófico pouco profundo, textura argilosa, suavemente ondulado }+ \text { Latossolo Roxo } \\
\text { eutrófico, textura argilosa e muito argilosa. }\end{array}$ \\
\hline $\mathrm{Pea}^{4}$ & $\begin{array}{l}\text { Podzólico Vermelho-Escuro álico, argila de baixa atividade, textura arenosa horizonte A, franca horizonte } \\
\text { B, suavemente ondulado + Latossolo Vermelho-Escuro álico textura franca, plano e levemente ondulado. }\end{array}$ \\
\hline & + significa presença de solo subdominante \\
\hline
\end{tabular}

${ }^{1}$ Neossolos Quartzarênicos; ${ }^{2}$ Gleissolos; ${ }^{3}$ Latossolos; ${ }^{4}$ Argissolos (Embrapa, 1999). 


\section{Procedimento metodológico}

O trabalho foi realizado em duas etapas. Na primeira etapa, por meio de revisão bibliográfica, pesquisaram-se as exigências edafoclimáticas das quatro espécies de eucalipto em estudo (Tabela 3). Além disso, consideraram-se também as áreas de preservação permanente (Lei 4.771/65), estabelecendose assim os primeiros parâmetros para o zoneamento de aptidão.

Tabela 3. Resumo das exigências edafoclimáticas de diversas espécies de eucalipto, segundo a literatura. Table 3. Summary of edafoclimatic needs for some species of eucalyptus according to literature.

\begin{tabular}{|c|c|c|c|c|}
\hline & E. camaldulensis & E. citriodora & E. grandis & E. urophylla \\
\hline $\begin{array}{l}\text { Temperatura } \\
\text { média }\left({ }^{\circ} \mathrm{C}\right)\end{array}$ & 17 a $23^{(1)}$ & 20 a $23^{(1)}$ & 17 a $23^{(1)}$ & $\begin{array}{c}19 \text { a } 24^{(1)} \\
20 \text { e } 30^{\text {o }(9)}\end{array}$ \\
\hline $\begin{array}{l}\text { Temperatura } \\
\text { máxima }\left({ }^{\circ} \mathrm{C}\right)\end{array}$ & $\begin{array}{l}16 \text { a } 40^{(1)} \\
27 \text { a } 40^{(5)}\end{array}$ & $\begin{array}{c}30 \text { a } 36^{(5)} \\
21,5 \text { a } 40^{(1)}\end{array}$ & $\begin{array}{l}20 \text { a } 32^{(1)} \\
24 \text { a } 32^{(5)}\end{array}$ & 21 a $29^{(1)}$ \\
\hline $\begin{array}{l}\text { Temperatura } \\
\text { mínima }\left({ }^{\circ} \mathrm{C}\right)\end{array}$ & $\begin{array}{c}-5 \text { a } 20^{(1)} \\
3 \text { a } 15^{(5)} \\
\text { suporta geadas leves }^{(3)}\end{array}$ & $\begin{array}{c}5 \text { a } 12,0^{(5)} \\
0 \text { a } 19,5^{(1)} \\
\text { suporta geadas leves }^{(3)}\end{array}$ & $\begin{array}{c}-5 \text { a } 19^{(1)} \\
3 \text { a } 17^{(5)} \\
\text { reg. livres de geadas } \\
\text { severas }^{(3)(4)}\end{array}$ & $\begin{array}{l}8 \text { a } 20^{(1)} \\
\text { reg. livres geadas } \\
\text { severas }^{(3)(4)}\end{array}$ \\
\hline $\begin{array}{l}\text { Precipitação } \\
(\mathrm{mm})\end{array}$ & $\begin{array}{l}250 \text { a } 1800^{(1)} \\
150 \text { a } 1250^{(5)}\end{array}$ & $\begin{array}{l}650 \text { a } 1600^{(5)} \\
350 \text { a } 1800^{(1)}\end{array}$ & $\begin{array}{c}550 \text { a } 1800^{(1)} \\
1000 \text { a } 3500^{(5)}\end{array}$ & $\begin{array}{l}900 \text { a } 1800^{(1)} \\
600 \text { a } 2.500^{(9)}\end{array}$ \\
\hline $\begin{array}{l}\text { Déficit hídrico } \\
(\mathrm{mm})\end{array}$ & $\begin{array}{l}0 \text { a } 90^{(1)} \\
\text { recom. para regiões de } \\
\text { déficit híd. anual elevado }{ }^{(4)}\end{array}$ & 30 a $90^{(1)}$ & $\begin{array}{c}0 \text { a } 120^{(1)} \text { suporta período } \\
\text { de seca inferior. a } 3 \\
\text { meses }^{(3)}\end{array}$ & $\begin{array}{c}30 \text { a } 210^{(1)} \\
\text { períodos } \\
\text { marcantes de } \\
\text { deficiência } \\
\text { hídrica }^{(9)}\end{array}$ \\
\hline Latitude & $12,5^{\circ} \mathrm{S}$ a $38^{\circ} \mathrm{S}^{(5)}$ & $16^{\circ} 45^{\prime} \mathrm{S}$ a $26^{\circ} \mathrm{S}^{(5)}$ & $16^{\circ} \mathrm{S}$ a $33^{\circ} \mathrm{S}^{(5)}$ & $7,5^{\circ} \mathrm{S}$ a $10^{\circ} \mathrm{S}^{(9)}$ \\
\hline Altitude (m) & 20 a $700 \mathrm{~m}^{(5)}$ & 70 a $1.000 \mathrm{~m}^{(5)}$ & $\begin{array}{l}0 \text { a } 900 \mathrm{~m}^{(8)} \\
0 \text { a } 1100 \mathrm{~m}^{(5)} \\
0 \text { a } 1250 \mathrm{~m}^{(2)}\end{array}$ & 300 a $3.000 \mathrm{~m}^{(9)(2)}$ \\
\hline
\end{tabular}

$\mathrm{Na}$ segunda etapa, foram considerados os níveis de manejo, cujas análises se basearam nos "fatores limites para cada unidade de solo e níveis de manejo" (Tabela 4) extraídos do trabalho de Macchetta (2004).

Tabela 4. Fatores limites para cada unidade de solo e níveis de manejo com base no Sistema FAO/Brasileiro.

Table 4. Limiting factors for each soil class and management levels basing on the FAO/Brazilian System

\begin{tabular}{|c|c|c|c|c|c|c|c|c|c|c|c|c|c|c|c|}
\hline \multirow{2}{*}{$\begin{array}{l}\text { Unidade } \\
\text { de solo }\end{array}$} & \multicolumn{3}{|c|}{$\Delta \mathbf{N}$} & \multicolumn{3}{|c|}{$\Delta \mathbf{A}$} & \multicolumn{3}{|c|}{$\Delta \mathbf{O}$} & \multicolumn{3}{|c|}{$\Delta \mathbf{E}$} & \multicolumn{3}{|c|}{$\Delta \mathbf{M}$} \\
\hline & $\mathbf{A}$ & B & $\mathrm{C}$ & $\mathbf{A}$ & B & $\mathrm{C}$ & $\mathbf{A}$ & B & $\mathbf{C}$ & $\mathbf{A}$ & B & $\mathrm{C}$ & $\mathbf{A}$ & B & $\mathrm{C}$ \\
\hline AQa1 & $\mathrm{F}$ & $\mathrm{F}$ & $\mathrm{M} / \mathrm{F} \underline{2}$ & $\mathrm{M}$ & $\mathrm{M}$ & $\mathrm{M}$ & $\mathrm{N}$ & $\mathrm{N}$ & $\mathrm{N}$ & $\mathrm{L}$ & $\mathrm{N} / \mathrm{L} \underline{1}$ & $\mathrm{~N} / \mathrm{L} 1$ & $\mathrm{~N}$ & $\mathrm{~N}$ & $\mathrm{~N}$ \\
\hline HGPe7 & $\mathrm{L} / \mathrm{M}$ & $\mathrm{L} \underline{1}$ & $\mathrm{~N} \underline{2}$ & $\mathrm{~N}$ & $\mathrm{~N}$ & $\mathrm{~N}$ & $\mathrm{M} / \mathrm{F}$ & $\mathrm{M} / \mathrm{F}$ & $\mathrm{M} 2$ & $\mathrm{~N}$ & $\mathrm{~N}$ & $\mathrm{~N}$ & $\mathrm{~F}$ & $\mathrm{~F}$ & $\mathrm{~F} / \mathrm{M} 2$ \\
\hline LEa2 & $\mathrm{F}$ & M1 & $\mathrm{L} \underline{2}$ & $\mathrm{~L} / \mathrm{M}$ & $\mathrm{L} / \mathrm{M}$ & $\mathrm{L} / \mathrm{M}$ & $\mathrm{N}$ & $\mathrm{N}$ & $\mathrm{N}$ & $\mathrm{L}$ & $\mathrm{N} / \mathrm{L} \underline{1}$ & $\mathrm{~N} / \mathrm{L} 1$ & $\mathrm{~N}$ & $\mathrm{~N}$ & $\mathrm{~N}$ \\
\hline LEa4 & $\mathrm{F}$ & $\mathrm{M} \underline{1}$ & $\mathrm{~L} / \mathrm{M} \underline{2}$ & $\mathrm{~L}$ & $\mathrm{~L}$ & $\mathrm{~L}$ & $\mathrm{~N}$ & $\mathrm{~N}$ & $\mathrm{~N}$ & $\mathrm{~L} / \mathrm{M}$ & $\mathrm{L} 1$ & $\mathrm{~L} 1$ & $\mathrm{~N}$ & $\mathrm{~N}$ & $\mathrm{~N}$ \\
\hline LEa11 & $\mathrm{F}$ & $\mathrm{M} \underline{1}$ & $\mathrm{~L} / \mathrm{M} \underline{2}$ & $\mathrm{M}$ & $\mathrm{M}$ & $\mathrm{M}$ & $\mathrm{N}$ & $\mathrm{N}$ & $\mathrm{N}$ & $\mathrm{L} / \mathrm{M}$ & $\mathrm{L} \underline{1}$ & $\mathrm{~L} 1$ & $\mathrm{~N}$ & $\mathrm{~N}$ & $\mathrm{~N}$ \\
\hline LEa15 & \multicolumn{15}{|c|}{ Não determinado } \\
\hline LEa20 & $\mathrm{F}$ & M1 & $\mathrm{L} / \mathrm{M} \underline{2}$ & M & M & M & $\mathrm{N}$ & $\mathrm{N}$ & $\mathrm{N}$ & $\mathrm{L} / \mathrm{M}$ & $\mathrm{L} 1$ & $\mathrm{~L} 1$ & $\mathrm{~N}$ & $\mathrm{~N}$ & $\mathrm{~N}$ \\
\hline LRa1 & $\mathrm{M} / \mathrm{F}$ & M1 & $\mathrm{L} \underline{2}$ & $\mathrm{~L} / \mathrm{M}$ & $\mathrm{L} / \mathrm{M}$ & $\mathrm{L} / \mathrm{M}$ & $\mathrm{N}$ & $\mathrm{N}$ & $\mathrm{N}$ & $\mathrm{N} / \mathrm{L}$ & $\mathrm{N}$ & $\mathrm{N}$ & $\mathrm{N}$ & $\mathrm{N}$ & $\mathrm{N}$ \\
\hline LRa2 & $\mathrm{M} / \mathrm{F}$ & M 1 & $\mathrm{~L} \underline{2}$ & $\mathrm{~L}$ & $\mathrm{~L}$ & $\mathrm{~L}$ & $\mathrm{~N}$ & $\mathrm{~N}$ & $\mathrm{~N}$ & $\mathrm{~L}$ & $\mathrm{~N} / \mathrm{L} \underline{1}$ & $\mathrm{~N} / \mathrm{L} 1$ & $\mathrm{~N}$ & $\mathrm{~N}$ & $\mathrm{~N}$ \\
\hline LRd5 & $\mathrm{M} / \mathrm{F}$ & $\mathrm{M} \underline{1}$ & $\mathrm{~L} \underline{2}$ & $\mathrm{~L} / \mathrm{M}$ & $\mathrm{L} / \mathrm{M}$ & $\mathrm{L} / \mathrm{M}$ & $\mathrm{N}$ & $\mathrm{N}$ & $\mathrm{N}$ & $\mathrm{L}$ & $\mathrm{N} / \mathrm{L} \underline{1}$ & $\mathrm{~N} / \mathrm{L} 1$ & $\mathrm{~N} / \mathrm{L}$ & $\mathrm{N} / \mathrm{L}$ & $\mathrm{N} / \mathrm{L}$ \\
\hline LRd7 & $\mathrm{M} / \mathrm{F}$ & $\mathrm{M} \underline{1}$ & $\mathrm{~L} \underline{2}$ & $\mathrm{~L}$ & $\mathrm{~L}$ & $\mathrm{~L}$ & $\mathrm{~N}$ & $\mathrm{~N}$ & $\mathrm{~N}$ & $\mathrm{~L}$ & $\mathrm{~N} / \mathrm{L} \underline{1}$ & $\mathrm{~N} / \mathrm{L} 1$ & $\mathrm{~N}$ & $\mathrm{~N}$ & $\mathrm{~N}$ \\
\hline LRe1 & $\mathrm{N} / \mathrm{L}$ & $\mathrm{N} / \mathrm{L} 1$ & $\mathrm{~N} \underline{2}$ & $\mathrm{~L}$ & $\mathrm{~L}$ & $\mathrm{~L}$ & $\mathrm{~N}$ & $\mathrm{~N}$ & $\mathrm{~N}$ & $\mathrm{~L}$ & $\mathrm{~N} / \mathrm{L} \underline{1}$ & $\mathrm{~N} 1$ & $\mathrm{~N}$ & $\mathrm{~N}$ & $\mathrm{~N}$ \\
\hline Pea & $\mathrm{F}$ & M 1 & $\mathrm{~L} / \mathrm{M} \underline{2}$ & $\mathrm{M}$ & $\mathrm{M}$ & $\mathrm{M}$ & $\mathrm{N}$ & $\mathrm{N}$ & $\mathrm{N}$ & $\mathrm{L} / \mathrm{M}$ & $\mathrm{L} \underline{1}$ & $\mathrm{~N} / \mathrm{L} \underline{1}$ & $\mathrm{~N}$ & $\mathrm{~N}$ & $\mathrm{~N}$ \\
\hline
\end{tabular}

Ligeiro (L); Ligeiro/Moderado (L/M); Moderado (M); Moderado/Forte (M/F); Forte (F). Fonte: modificado de Maccheta, 2004. 
Com a utilização do banco de dados resultante do trabalho de Daniel et al. (2002) e literatura consultada, foi implementada uma nova base de dados com o uso do software SPRING versão 4.1, da seguinte forma: a) criação do banco de dados; b) definição do projeto, estabelecendo-se os limites geográficos da área de estudo e a projeção cartográfica (UTM/Córrego Alegre), escala 1:250.000; c) definição do esquema conceitual, especificando-se as Categorias com seus respectivos Modelos e nomeação dos Planos de Informação (PI's); d) geração dos mapas de aptidão e manejo, através do cruzamento dos PI's, utilizando-se da linguagem de programação do próprio SPRING: LEGAL Linguagem Espacial para Geoprocessamento Algébrico.

Na primeira etapa, optou-se pela escolha de dois níveis de aptidão: apta (A) e restrita (R). O nível (A) são áreas com condições edafoclimáticas favoráveis para o bom desenvolvimento e produção da cultura em escala econômica; o nível $(\mathrm{R})$ são áreas com inviabilidade do cultivo por um ou mais dos fatores aqui considerados.

O PI altimetria, importado de Daniel et al. (2002), deu origem aos mapas de altitude e declividade. A partir da hidrografia, também importada dos mesmos autores, foi gerado o mapa de áreas de preservação permanente ao longo dos cursos d'água, estabelecendo-se, a princípio, duas classes $(30 \mathrm{~m}$ e $50 \mathrm{~m})$ a partir do Código Florestal, tendo em vista que dentro da bacia em estudo a largura dos rios não ultrapassa o limite de 50 $\mathrm{m}$. Em seguida, foram unidas formando uma só classe. Os pontos aproximados de mudança entre as classes "30 m" e "50 m" ao longo dos rios foram determinados em visitas a campo e em entrevistas com moradores locais.

$\mathrm{Na}$ Categoria "pedologia" foram consideradas como aptas as classes dos Latossolos, Argissolos e Neossolos Quartzarênicos, e como restritas, os Gleissolos, que, pelas suas características (nível piezométrico elevado do aqüífero superficial e facilidade de inundação), não são indicados para o plantio. Da mesma forma, a Categoria "uso da terra" reduziu-se às classes apta (Agricultura, Capoeira, Cerrado, Eucalipto, Mata e Pastagem) e restrita (Complexo de vegetações, Complexo urbano e Corpos d'água).

Definidas as Categorias e respectivos PI's, procedeu-se à elaboração da tabela 5, situando as restrições relacionadas a cada espécie de eucalipto, para posterior geração dos mapas de aptidão.

Para a segunda etapa, considerou-se o método FAO-brasileiro (RAMALHO FILHO; BEEK, 1995), segundo o qual o nível de manejo "B” seria o recomendado para silvicultura.

Em função disso, para a geração do mapa dos níveis de manejo reclassificaram-se os fatores limites para cada unidade de solo e níveis de manejo (Tabela 4) do trabalho de Macchetta (2004), resultando em pontuações diferenciadas para cada unidade de solo (Tabela 6). Foram utilizadas 7 (sete) classes de extratificação, conforme o crescente nível de dificuldade e necessidade de input tecnológico: Boa (B); Boa/Moderada (B/M); Moderada (M); Moderada/Regular (M/R); Regular (R); Regular/Restrita $(\mathrm{R} / \mathrm{R})$ e Restrita $(\mathrm{Rt})$. Em seguida, considerando as restrições comuns às quatro espécies de eucalipto (Tabela 5) e a reclassificação dos fatores limites para cada unidade de solo e níveis de manejo (Tabela 6), foi gerado o mapa dos níveis de manejo.

\section{RESULTADOS E DISCUSSÃO}

Com base nas restrições apresentadas (Tabela 5), os Planos de Informação foram reclassificados, gerando matrizes com resolução 15 x $15 \mathrm{~m}$. O processamento do programa em LEGAL considerou todas as combinações possíveis entre as Classes dos PI's gerando dois mapas: mapa de aptidão para $E$. camaldulensis, E. citriodora e E. grandis (Figura 3), e mapa de aptidão para E. urophylla (Figura 4). Da mesma forma, considerando as informações das tabelas 5 e 6, foi gerado o mapa dos níveis de manejo (Figura 4) e calculadas as áreas de cada classe considerada (Tabela 9).

A análise da figura 3 permite observar que a maior parte da bacia do rio Dourados $(88,67 \%)$ encontra-se apta ao cultivo das espécies considerando-se as restrições estabelecidas (Tabela 5), corroborando o trabalho de Golfari (1978) com relação ao E. grandis. A maior parte das regiões consideradas com restrições $(11,33 \%)$ está relacionada com as áreas de preservação permanente, ao longo dos cursos d'água ou próximos a eles (Tabela 7).

O E. urophylla foi a única espécie a apresentar diferença de restrições, especificamente com relação à altitude. Dados da literatura demonstram que a área de abrangência dessa espécie no local de origem se encontra em altitudes de $300 \mathrm{~m}$ a $3.000 \mathrm{~m}$ (PAYNTON, 1979; GOLFARI, 1978). O Mapa de Aptidão para E. urophylla (Figura 4) apresentou 87,3\% para a classe "apta", também corroborando o trabalho de Golfari (1978), e 12,7\% para a classe "restrita" (Tabela 8). 
Tabela 5. Restrições consideradas para o cruzamento dos PI's e geração dos mapas de aptidão.

Table 5. Restrictions considered for cross-referencing of information layers and generation of land aptitude maps.

\begin{tabular}{|c|c|c|c|c|c|c|c|c|c|}
\hline \multicolumn{2}{|l|}{ Restrições ** } & \multicolumn{2}{|c|}{ E. camaldulensis } & \multicolumn{2}{|c|}{ E. citriodora } & \multicolumn{2}{|c|}{ E. grandis } & \multicolumn{2}{|c|}{ E. urophylla } \\
\hline PI's & $\begin{array}{l}\text { Aptidão } \\
\text { Classes }\end{array}$ & A & $\mathrm{R}$ & A & $\mathrm{R}$ & A & $\mathrm{R}$ & A & $\mathrm{R}$ \\
\hline \multirow{2}{*}{ Altitude } & 0 a $300 \mathrm{~m}$ & $\mathrm{x}$ & & $\mathrm{x}$ & & $\mathrm{x}$ & & & $\mathrm{x}$ \\
\hline & $>300 \mathrm{~m}$ & $\mathrm{x}$ & & $\mathrm{x}$ & & $\mathrm{x}$ & & $\mathrm{x}$ & \\
\hline \multirow{2}{*}{ Buffer* } & 0 a $30 \mathrm{~m}$ & & $\mathrm{x}$ & & $\mathrm{x}$ & & $\mathrm{x}$ & & $\mathrm{x}$ \\
\hline & 0 a $50 \mathrm{~m}$ & & $\mathrm{x}$ & & $\mathrm{x}$ & & $\mathrm{x}$ & & $\mathrm{x}$ \\
\hline \multirow{2}{*}{ Declividade } & 0 a $100 \%$ & $\mathrm{x}$ & & $\mathrm{x}$ & & $\mathrm{x}$ & & $\mathrm{x}$ & \\
\hline & $>100 \%$ & & $\mathrm{x}$ & & $\mathrm{x}$ & & $\mathrm{x}$ & & $\mathrm{x}$ \\
\hline \multirow{4}{*}{ Solos } & Argissolos & $\mathrm{x}$ & & $\mathrm{x}$ & & $\mathrm{x}$ & & $\mathrm{x}$ & \\
\hline & Neossolos & $\mathrm{x}$ & & $\mathrm{x}$ & & $\mathrm{x}$ & & $\mathrm{x}$ & \\
\hline & Gleissolos & & $\mathrm{x}$ & & $\mathrm{x}$ & & $\mathrm{x}$ & & $\mathrm{x}$ \\
\hline & Latossolos & $\mathrm{x}$ & & $\mathrm{x}$ & & $\mathrm{x}$ & & $\mathrm{x}$ & \\
\hline \multirow{9}{*}{ Uso da terra } & Agricultura $^{6}$ & $\mathrm{x}$ & & $\mathrm{x}$ & & $\mathrm{x}$ & & $\mathrm{x}$ & \\
\hline & Capoeira $^{3}$ & $\mathrm{x}$ & & $\mathrm{x}$ & & $\mathrm{x}$ & & $\mathrm{x}$ & \\
\hline & Cerrado $^{2}$ & $\mathrm{x}$ & & $\mathrm{x}$ & & $\mathrm{x}$ & & $\mathrm{x}$ & \\
\hline & Complexo de vegetações ${ }^{8}$ & & $\mathrm{x}$ & & $\mathrm{x}$ & & $\mathrm{X}$ & & $\mathrm{x}$ \\
\hline & Complexo urbano 9 & & $\mathrm{x}$ & & $\mathrm{x}$ & & $\mathrm{x}$ & & $\mathrm{x}$ \\
\hline & Corpos d'água ${ }^{7}$ & & $\mathrm{x}$ & & $\mathrm{x}$ & & $\mathrm{x}$ & & $\mathrm{x}$ \\
\hline & Eucalipto $^{4}$ & $\mathrm{x}$ & & $\mathrm{x}$ & & $\mathrm{x}$ & & $\mathrm{x}$ & \\
\hline & Mata $^{1}$ & $\mathrm{x}$ & & $\mathrm{x}$ & & $\mathrm{x}$ & & $\mathrm{x}$ & \\
\hline & Pastagem $^{5}$ & $\mathrm{x}$ & & $\mathrm{x}$ & & $\mathrm{x}$ & & $\mathrm{x}$ & \\
\hline
\end{tabular}

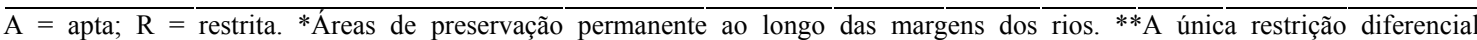
considerada foi a de E. urophylla, abaixo de $300 \mathrm{~m}$. ' matas: pequenos fragmentos, remanescentes de floresta semidecídua existentes na região, os quais encontram-se isolados, podendo ter algum grau de antropização; ${ }^{2}$ cerrado: pequenos fragmentos remanescentes dessa tipologia florestal existentes na região; ${ }^{3}$ capoeira: pequenos fragmentos remanescentes das duas tipologias anteriores, com alto grau de antropização; ${ }^{4}$ eucalipto: áreas cobertas pelo gênero Eucalyptus, normalmente de pequena extensão e em alguns casos em estreitas faixas às margens de estradas; ${ }^{5}$ pastagem: tipologia representada por pastos cultivados com espécies forrageiras, em geral utilizados na criação de bovinos, degradados ou não, predominantemente herbáceos mas podendo ocorrer arbustos e arvoretas invasoras no estrato lenhoso. As espécies comumente encontradas pertencem aos gêneros Brachiaria e Panicum; ${ }^{6}$ agricultura: cobertura vegetal composta normalmente por soja, milho e aveia; ${ }^{7}$ corpos d'água: categoria representada por espelhos d'água de qualquer natureza; ${ }^{8}$ complexo de vegetações: áreas formadas por complexa mistura de cerrado - mata, gramíneas nativas, vegetação normalmente associada a áreas de solos hidromórficos, em geral próximas às margens dos cursos d'água. A separação interna das diversas tipologias ali existentes é muito complexa para o nível de precisão proporcionado pela imagem LANDSAT; ${ }^{9}$ complexo urbano: unidades com concentrações de edificações, caracterizando vilas e cidades.

Tabela 6. Fatores limites para cada unidade de solo e níveis de manejo reclassificados.

Table 6. Reclassified limiting factors for each soil class and management levels.

\begin{tabular}{llccccc}
\hline Classe & Solo & Nutriente & Água & Oxigênio & Erosão & Mecanização \\
\hline $\mathrm{B}=2$ & $\mathrm{LRe} 1$ & $\mathrm{~N} / \mathrm{L} \underline{1}$ & $\mathrm{~L}$ & $\mathrm{~N}$ & $\mathrm{~N} / \mathrm{L} \underline{1}$ & $\mathrm{~N}$ \\
$\mathrm{~B} / \mathrm{M}=3,5$ & $\mathrm{LRa} 2$ & $\mathrm{M} \underline{1}$ & $\mathrm{~L}$ & $\mathrm{~N}$ & $\mathrm{~N} / \mathrm{L} \underline{1}$ & $\mathrm{~N}$ \\
$\mathrm{~B} / \mathrm{M}=3,5$ & $\mathrm{LRd} 7$ & $\mathrm{M} \underline{1}$ & $\mathrm{~L}$ & $\mathrm{~N}$ & $\mathrm{~N} / \mathrm{L} \underline{1}$ & $\mathrm{~N}$ \\
$\mathrm{~B} / \mathrm{M}=3,5$ & $\mathrm{LRa} 1$ & $\mathrm{M} \underline{1}$ & $\mathrm{~L} / \mathrm{M}$ & $\mathrm{N}$ & $\mathrm{N}$ & $\mathrm{N}$ \\
$\mathrm{M}=4$ & $\mathrm{LEa} 4$ & $\mathrm{M} \underline{1}$ & $\mathrm{~L}$ & $\mathrm{~N}$ & $\mathrm{~L} \underline{1}$ & $\mathrm{~N}$ \\
$\mathrm{M}=4$ & $\mathrm{LEa} 2$ & $\mathrm{M} \underline{\mathrm{1}}$ & $\mathrm{L} / \mathrm{M}$ & $\mathrm{N}$ & $\mathrm{N} / \mathrm{L} \underline{1}$ & $\mathrm{~N}$ \\
$\mathrm{M} / \mathrm{R}=4,5$ & $\mathrm{LRd5}$ & $\mathrm{M} \underline{1}$ & $\mathrm{~L} / \mathrm{M}$ & $\mathrm{N}$ & $\mathrm{N} / \mathrm{L} \underline{1}$ & $\mathrm{~N} / \mathrm{L}$ \\
$\mathrm{R}=5$ & $\mathrm{LEa} 11$ & $\mathrm{M} \underline{1}$ & $\mathrm{M}$ & $\mathrm{N}$ & $\mathrm{L} \underline{1}$ & $\mathrm{~N}$ \\
$\mathrm{R}=5$ & $\mathrm{LEa} 20$ & $\mathrm{M} \underline{1}$ & $\mathrm{M}$ & $\mathrm{N}$ & $\mathrm{L} \underline{1}$ & $\mathrm{~N}$ \\
$\mathrm{R}=5$ & $\mathrm{Pea}$ & $\mathrm{M} \underline{1}$ & $\mathrm{M}$ & $\mathrm{N}$ & $\mathrm{L} \underline{1}$ & $\mathrm{~N}$ \\
$\mathrm{R} / \mathrm{R}=5,5$ & $\mathrm{AQa} 1$ & $\mathrm{~F}$ & $\mathrm{M}$ & $\mathrm{N}$ & $\mathrm{N} / \mathrm{L} \underline{1}$ & $\mathrm{~N}$ \\
$\mathrm{Rt}=6,5$ & $\mathrm{HGPe} 7$ & $\mathrm{~L} \underline{\mathrm{N}}$ & $\mathrm{N}$ & $\mathrm{M} / \mathrm{F}$ & $\mathrm{N}$ & $\mathrm{F}$ \\
\hline
\end{tabular}

Pontuação considerada para cada fator limitante: Nulo $(\mathrm{N})=0 ;$ Nulo/Ligeiro $(\mathrm{N} / \mathrm{L})=0,5 ;$ Ligeiro $(\mathrm{L})=1 ;$ Ligeiro/Moderado $(\mathrm{L} / \mathrm{M})$ $=1,5 ;$ Moderado $(\mathrm{M})=2 ;$ Moderado/Forte $(\mathrm{M} / \mathrm{F})=2,5 ;$ Forte $(\mathrm{F})=3$. 


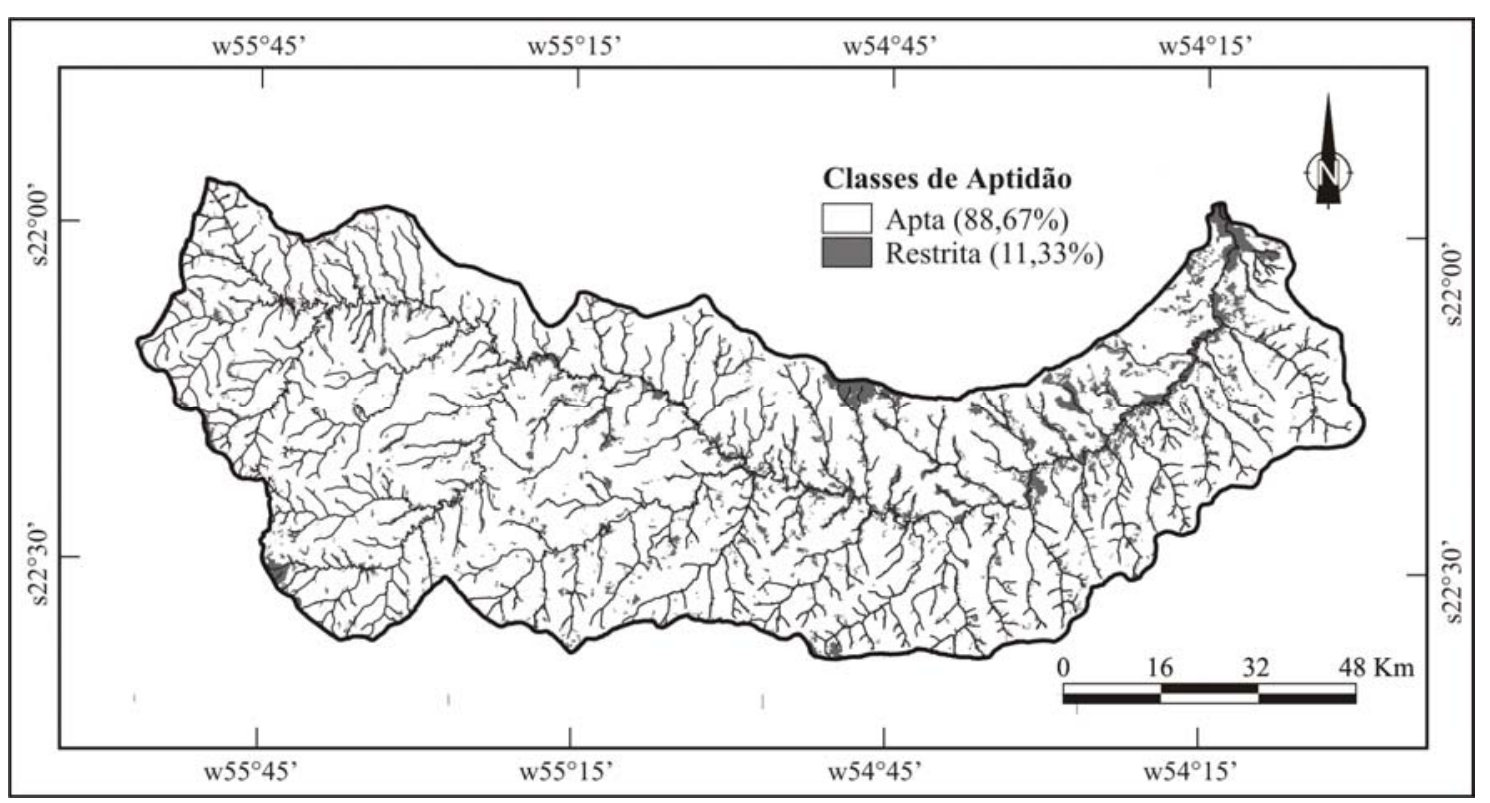

Figura 3. Mapa de aptidão para E. camaldulensis, E. citriodora e E. grandis na bacia hidrográfica do rio Dourados, MS.

Figure 3. Land aptitude map for E. camaldulensis, E. citriodora and E. grandis in Dourados River's Basin, MS.

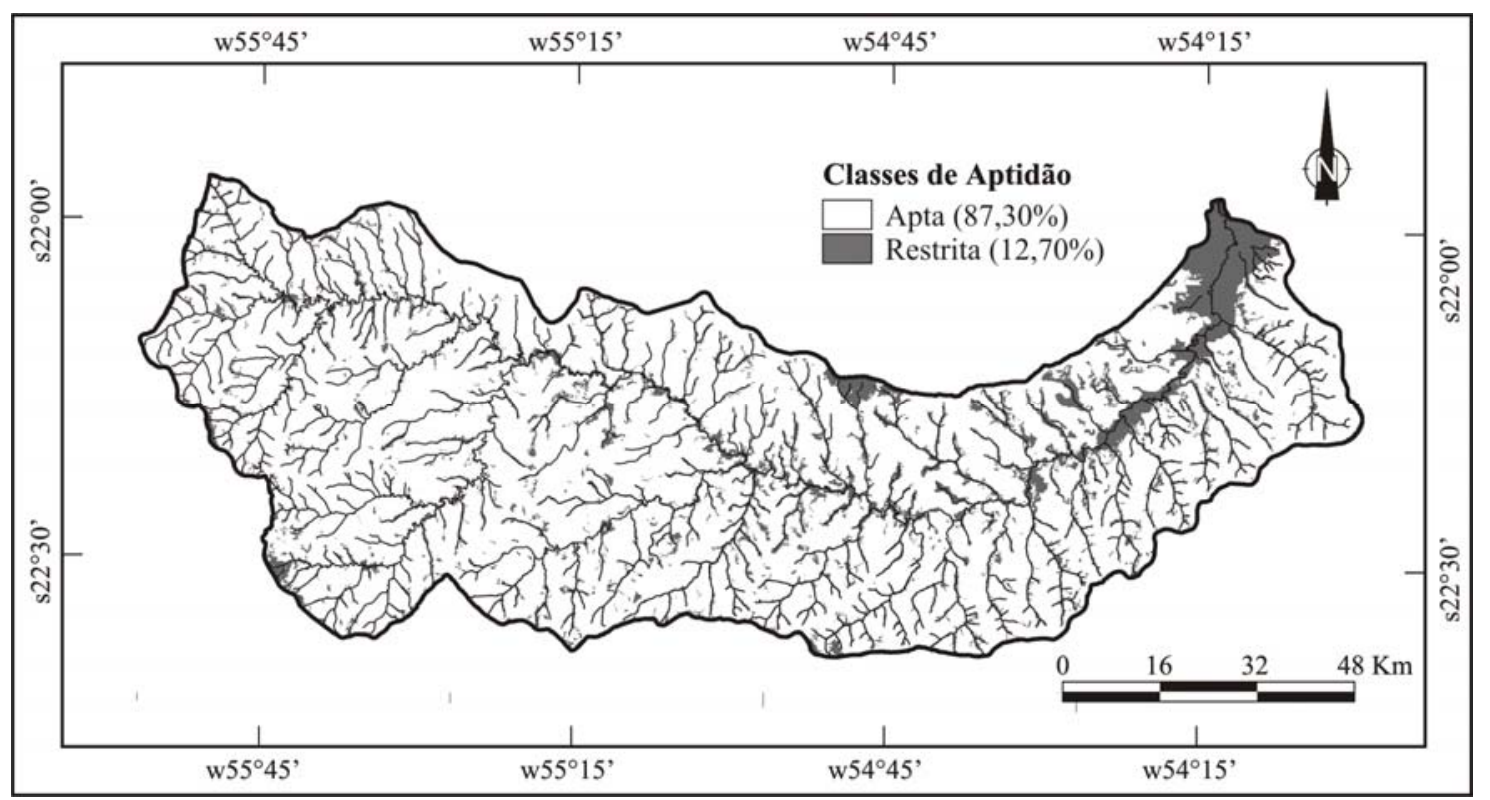

Figura 4. Mapa de aptidão para E. urophylla na bacia hidrográfica do rio Dourados, MS.

Figure 4. Land aptitude map for E. urophylla in Dourados River's Basin, MS. 
Tabela 7. Área das classes de aptidão para E. camaldulensis, E. citriodora e E. grandis.

Table 7. Area for land aptitude class for E. camaldulensis, E. citriodora and E. grandis.

\begin{tabular}{lcc}
\hline Classes & Área $\left(\mathbf{k m}^{\mathbf{2}}\right)$ & $\mathbf{\%}$ \\
\hline Apta & $8.198,908$ & 88,67 \\
Restrita & $1.039,685$ & 11,33 \\
Total & $9.238,593$ & 100,00 \\
\hline
\end{tabular}

Tabela 8. Área das classes de aptidão para E. urophylla.

Table 8. Area for land aptitude classification of E. urophylla.

\begin{tabular}{lcc}
\hline Classes & Área $\left(\mathbf{k m}^{\mathbf{2}}\right)$ & $\mathbf{\%}$ \\
\hline Apta & $8.077,205$ & 87,30 \\
Restrita & $1.161,388$ & 12,70 \\
Total & $9.238,593$ & 100,00 \\
\hline
\end{tabular}

Um aspecto a ser destacado é a inclusão das classes de uso da terra Agricultura, Mata, Cerrado e Capoeira como áreas aptas para o cultivo do eucalipto. A primeira (Agricultura) concorre com os solos mais nobres da região, e as outras (Mata, Cerrado e Capoeira) concentram as últimas áreas residuais de vegetação nativa da bacia. Tratando-se de uma primeira aproximação e atendendo o objetivo deste trabalho, este zoneamento não contempla essas discussões, que estariam direcionadas a questões legais e ecológicas.

Com relação ao mapa das classes de manejo (Figura 5), de uma maneira geral as limitações dos solos da região se baseiam na fertilidade, sendo que as características que determinaram as áreas restritas estão relacionadas ao excesso de água ou deficiência de oxigênio e à dificuldade de mecanização.

Analisando a tabela 9, pode-se observar que um grande percentual dos solos da região $(65,95 \%)$ encontra-se nas classes consideradas boa e boa/moderada, sugerindo que, em termos de manejo para o eucalipto, não há maiores dificuldades para o seu cultivo nessas áreas. Num extremo $(0,89 \%)$, encontramse as áreas mais nobres da região, e noutro $(10,92 \%)$ estão as áreas com maior restrição, constituídas basicamente por Gleissolos e áreas de preservação permanente ao longo dos cursos d'água.

Tabela 9. Área total das classes de manejo consideradas.

Table 9. Total area of the considered management units.

\begin{tabular}{lrr}
\hline Classe & Área $\left(\mathbf{k m}^{\mathbf{2}} \mathbf{)}\right.$ & $\mathbf{\%}$ \\
\hline Boa & 82,386 & 0,89 \\
Boa/moderada & $6.011,016$ & 65,06 \\
Moderada & $1.280,644$ & 13,86 \\
Moderada/regular & 10,925 & 0,12 \\
Regular & 785,360 & 8,50 \\
Regular/restrita & 6,083 & 0,07 \\
Restrita & $1.008,985$ & 10,92 \\
Não determinada & 53,192 & 0,58 \\
Total da bacia & $9.238,594$ & 100,00 \\
\hline
\end{tabular}

Entre as unidades de solos encontradas na bacia, algumas não tiveram seus fatores limites (nutrientes, água, oxigênio, erosão e mecanização) determinados, por não haver uma descrição do referido perfil, sendo incluídas na classe "Não determinada".

Os mapas finais (Figuras 3, 4 e 5) caracterizam áreas potenciais para o cultivo das espécies de eucalipto consideradas na região de estudo, possibilitando ainda uma análise qualitativa dos níveis tecnológicos (manejo) a serem implementados. Deve-se lembrar, no entanto, a necessidade da observação de parâmetros ambientais adequados e a devida priorização das reais necessidades de uso da terra antes da decisão sobre o sistema a ser instalado. 


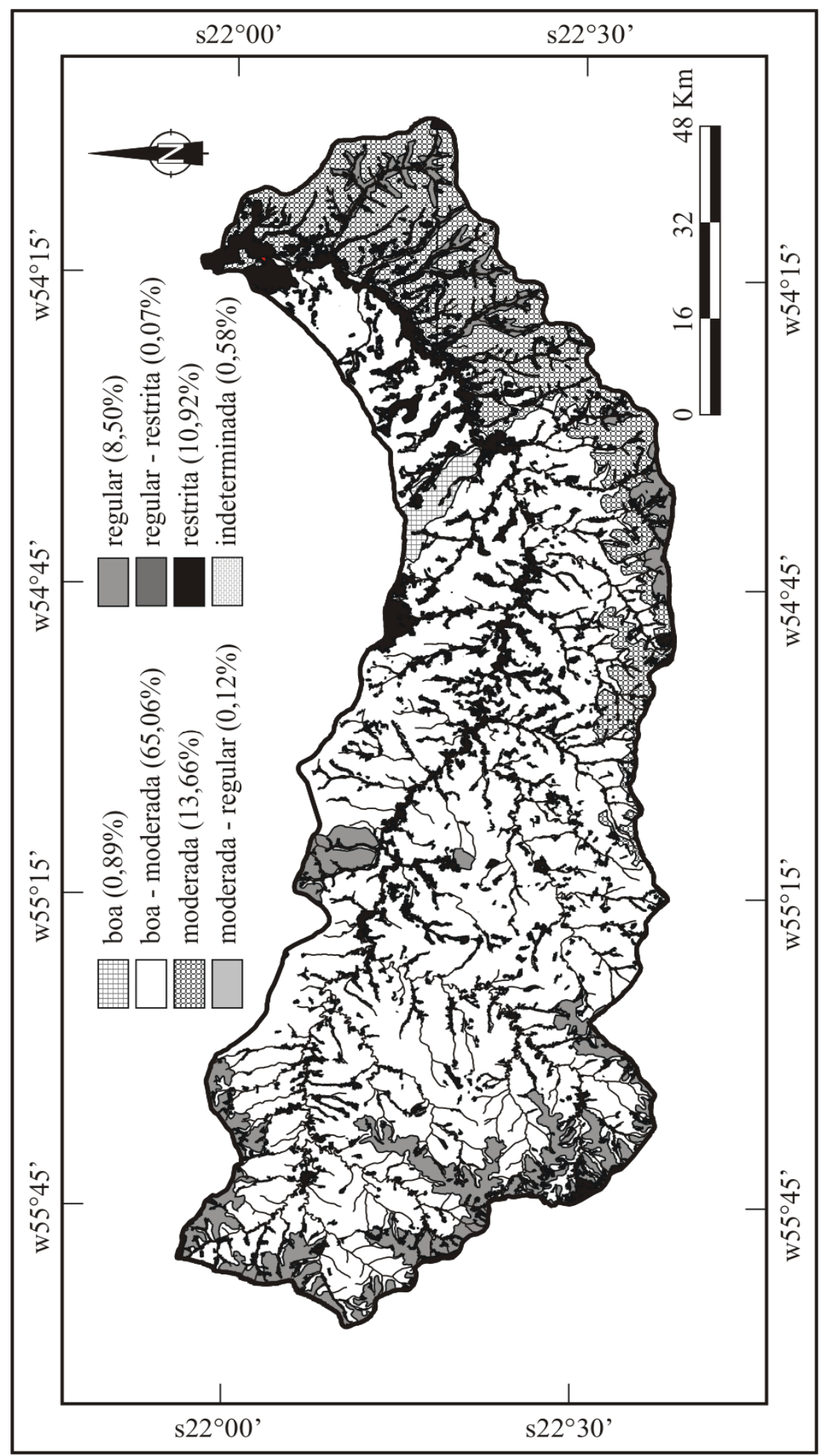

Figura 5. Mapa das classes de manejo considerados para silvicultura na bacia hidrográfica do rio Dourados, MS.

Figure 5. Considered map of management levels for silviculture in Dourados River's basin, MS. 


\section{CONCLUSÃO}

A utilização do software SPRING mostrou-se adequada para a geração de mapas de aptidão e manejo para espécies florestais. Apesar das limitações decorrentes do pouco detalhamento dos mapeamentos básicos (escala pequena) e da carência de dados meteorológicos dentro da área da bacia (poucas estações), o mapeamento foi satisfatório, evidenciando um grande potencial para o cultivo das espécies estudadas.

Este estudo representa um importante subsídio para a definição de políticas públicas voltadas para o gerenciamento e planejamento do uso do solo em escala regional. Contudo, para a implantação de ações pontuais, tais como o estímulo ao reflorestamento, faz-se necessário um maior nível de detalhamento, que por sua vez só será possível com base em mapeamentos básicos em escalas maiores e com a inclusão de estações meteorológicas na rede de monitoramento. A experimentação agronômica é outro ponto fundamental, uma vez que a região não dispõe de muitas informações sobre o comportamento e sistema de produção de espécies florestais na bacia.

A atual crise enfrentada pelo agronegócio local, com grande demanda de alternativas às culturas anuais tradicionais, em conjunto com o potencial apresentado por essa área, credencia a silvicultura como importante alternativa econômica para a região.

\section{REFERÊNCIAS}

BARROS, N. F.; NOVAIS, R. F.; CARDOSO, J. R.; MACEDO, P. R. Algumas relações solo-espécies de eucalipto em suas condições naturais. In: BARROS, N. F.; NOVAIS, R. F. Relação solo-eucalipto. Viçosa: Folha de Viçosa, 1990. 330p.

BOLAND, D. J.; BROOKER, M. I. H.; CHIPPENDALE, G. M.; HALL, N.; HYLAND, B. P. M.; JOHNSTON, R. D.; KLEINIG, D. A.; TURNER, J. D. Forest trees of Australia. 4.ed. Melbourne, Australia: CSIRO, 1994.

BRASIL. Lei 4.771, de 15 de setembro de 1965. Institui o novo Código Florestal. Disponível em: $<$ http://www.planalto.gov.br/ccivil_03/Leis/L4771.htm> Acesso em: 08 out. 2004.

CAMARA, G.; SOUZA, R. C. M.; FREITAS, U. M.; GARRIDO, J. SPRING: Integrating remote sensing and GIS by object-oriented data modelling. Computers and Graphics, New York, v.20, n.3, p.395-403, may/jun, 1996.

DANIEL, O. Teste de espécies / procedências de Eucalyptus spp em Dourados (MS), Brasil. Cerrados, Brasília, DF, v.1, n.1, p.24-27, 1998.

DANIEL, O.; VITORINO, A. C. T.; VERONESI, C. O.; QUEIROZ, L. S.; GELAIN, E. Mapeamento do uso da terra na bacia do rio dourados, MS, por meio de imagens Landsat, 2002. [S.1.]: DCA/UFMS. Relatório de Pesquisa não publicado.

EMBRAPA. Centro Nacional de Pesquisa de Solos (Rio de Janeiro, RJ). Sistema brasileiro de classificação de solos. Brasília, DF: Embrapa Produção de Informação, 1999. 412p.

FERREIRA, C. C. M. Zoneamento agroclimático para implantação de sistemas agroflorestais com eucaliptos em Minas Gerais. 158p. Dissertação (Mestrado em Meteorologia Agrícola) - Universidade Federal de Viçosa, Viçosa, MG, 1997.

FIETZ, C. R. Caracterização climática da região de Dourados visando à prática da irrigação. In: URCHEI, M. A.; FIETZ, C. R. Princípios de agricultura irrigada: caracterização e potencialidades em Mato Grosso do Sul. Dourados: Embrapa Agropecuária Oeste, 2001. p.69-76. (Documentos, n.37).

FINGER, C. A. G.; SCHUMACHER, M.V.; SCHNEIDER, P. R.; HOPPE, J. M. Influência da camada de impedimento no solo sobre o crescimento de Eucalyptus grandis (Hill) ex Maiden. Ciência Florestal, Santa Maria, v.6, n.1, p.137-145, 1996. 
GOLFARI, L.; CASER, R. L.; MOURA, V. P. G. Zoneamento ecológico esquemático para reflorestamento no Brasil ( $2^{\mathrm{a}}$. aproximação). Belo Horizonte: PNUD/FAO/IBDF/BRA-45, 1978. (Série Técnica n. 11).

HIGA, R. C. V.; MORA, A. L.; HIGA, A. R. Plantio de eucalipto na pequena propriedade rural. Colombo: Embrapa Florestas, 2000. 31p. (Embrapa Florestas. Documentos, n.54).

MACCHETTA, S. Verifica di cartografia pedológica del bacino del rio Dourados (Mato Grosso do Sul, Brasile). 96p. Monografia (Tesi di Laurea) - Università degli Studi di Torino, Torino, 2004.

MATO GROSSO DO SUL. Secretaria de Estado de Meio Ambiente. Fundação Estadual de Meio Ambiente Pantanal. Coordenadoria de Recursos Hídricos e qualidade Ambiental. Divisão Centro de Controle Ambiental. Microbacia hidrográfica do Rio Dourados: diagnóstico e implantação da rede básica de monitoramento da qualidade das águas. Campo Grande, MS, 2000. 78p.

PALUDZYSZYN FILHO, E. Indicações de Espécies para Plantio. In: Embrapa Florestas - Sistemas de Produção, n.4, 2002.

PAYNTON, R. J. Tree planting in Southern Africa: The Eucalyptus. Pretória: South Africa Forestry Research Institute, 1979. v.2, p.788-94.

RAMALHO FILHO, A.; BEEK, K. J. Sistema de avaliação da aptidão agrícola das terras. 3.ed. rev. Rio de Janeiro: Embrapa-CNPS, 1995. 65p.

RAMIRES JUNIOR, L. C. Programa estadual de desenvolvimento florestal de Mato Grosso do Sul. In: SEMINÁRIO SISTEMAS AGROFLORESTAIS E DESENVOLVIMENTO SUSTENTÁVEL, 2003, Campo Grande. Palestras... Campo Grande: EMBRAPA/CNPGC, 2003. 1 CD-ROM.

MATO GROSSO DO SUL. Secretaria de Estado de Planejamento e de Ciência e Tecnologia. Atlas multireferencial - Estado de Mato Grosso do Sul. Campo Grande, 1990. 2 p.

TOLEDO FILHO, D. V.; PIRES, C. L. S.; FERNANDES, P. S.; GARRIDO, M. A. O.; GIANOTTI, E.; ROSA, P. R. F. Competição entre algumas populações de Eucalyptus grandis Hill ex maiden. Boletim Técnico do Instituto Florestal, São Paulo, v.36, n.1, p.37-42, abr., 1982.

URCHEI, M. A. Potencial dos solos de Mato Grosso do Sul para agricultura irrigada. In: URCHEI, M. A.; FIETZ, C. R. Princípios de agricultura irrigada: caracterização e potencialidades em Mato Grosso do Sul. Dourados: Embrapa Agropecuária Oeste, 2001. 150p. p. 77-99. (Documentos, n.37). 